\title{
Balantidiasis in a Patient with Suspected Crohn's Disease
}

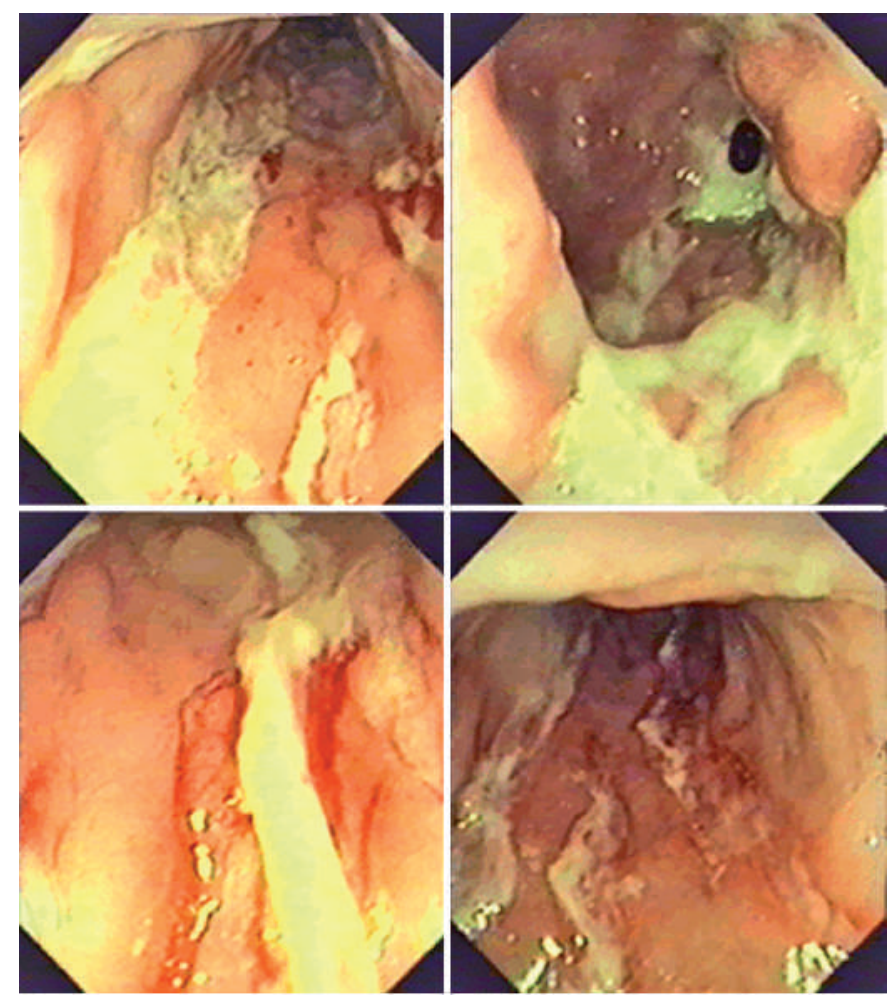

Figure 1 Colonoscopic appearance of the patient's large bowel.

A 15-year-old boy was referred to the gastroenterology department with a 2-month history of lower abdominal pain, intermittent diarrhea, fatigue, and weight loss. He had been well until 8 weeks previously, when he had developed watery diarrhea with six or seven bowel movements per day, cramps, headache, and fatigue. Tetracycline $0.6 \mathrm{~g}$ b.i.d. had been prescribed for suspected infectious diarrhea. The number of bowel movements decreased to three or four per day with the treatment, but the patient began to note blood in the feces. Detailed examination showed nothing but a body mass index of $17.9 \mathrm{~kg} / \mathrm{m}^{2}$, a temperature of $37.2^{\circ} \mathrm{C}$, mild tenderness across the lower abdomen without rebound, a positive guaiac test, mild iron-deficiency anemia, leukocytosis, and eosinophilia. Stool culture, ova, and parasites were negative. Colonoscopy revealed multiple long ulcers of various depths surrounded by congested mucosa; the diseased areas were separated by normal mucosa (Figure 1). Multiple biopsies were taken. The rectum and the part of the small intestine examined $(10 \mathrm{~cm})$ were not af- fected. Crohn's disease was suspected. On morphological examination, Balantidium coli was detected in the specimens taken from the ulcer margins (Figure 2). Initiation of treatment with metronidazole $0.5 \mathrm{~g}$ b.i.d. led to complete clinical recovery within 5 days. A colonoscopy checkup 1 month after admission showed that the colonic mucosa had healed. Nine months after the initial presentation, the clinical, laboratory and endoscopic examination revealed nothing but a few minor scars in the colon. The important part of the differential diagnosis of inflammatory bowel disease (IBD) from other inflammatory disorders is the endoscopic examination. Unfortunately, infectious and parasitic colitis may be not only clinically, but also endoscopically indistinguishable from IBD [1-3]. Stool culture and microscopy do not always make it possible to exclude the possibility of infectious colitis. A morphological examination is still the most reliable test for differentiating between infectious colitis and IBD [4].

Competing interests: None

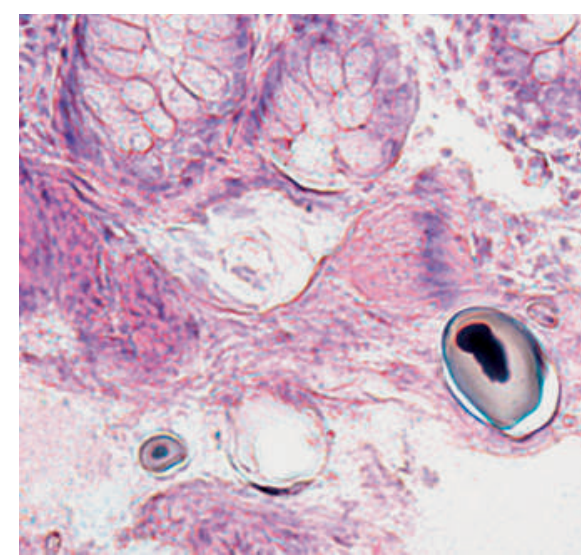

Figure 2 Balantidium coli in a specimen taken from an ulcer margin (hematoxylin-eosin, original magnification $\times 200$ )

Endoscopy_UCTN_Code_CCL_1AD_2AD

Endoscopy_UCTN_Code_CCL_1AD_2AZ

\section{Agapov}

Vladivostok Railway Hospital, Endoscopy department.

References

${ }^{1}$ Schumacher G. First attack of inflammatory bowel disease and infectious colitis: a clinical, histological and microbiological study with special reference to early diagnosis. Scand J Gastroenterol Suppl 1993; 198: 1 - 24 2 Patel AS, DeRidder PH. Amebic colitis masquerading as acute inflammatory bowel disease: the role of serology in its diagnosis. J Clin Gastroenterol 1989; 11: 407-410

${ }^{3}$ Tedesco FJ, Hardin RD, Harper RN et al. Infectious colitis endoscopically simulating inflammatory bowel disease: a prospective evaluation. Gastrointest Endosc 1983; 29: $195-197$

${ }^{4}$ Surawicz CM, Haggitt RC, Husseman M et al. Mucosal biopsy diagnosis of colitis: acute self-limited colitis and idiopathic inflammatory bowel disease. Gastroenterology 1994; 107: $755-763$

\section{Corresponding Author}

\section{Agapov, M.D.}

Vladivostok Railway Hospital, Endoscopy department

Verchne-portovaia 25

69003 Vladivostok, Russia

Fax: +7-4232-210227

E-mail: magapov@hotmail.com

Published online 3 March 2006

DOI: $10.1055 / \mathrm{s}-2006-925098$ 